\title{
Changes of High Sensitivity C-Reactive Protein During Clopidogrel Therapy in Patients Undergoing Percutaneous Coronary Intervention
}

\author{
Shokoufeh Hajsadeghi ${ }^{1}$; Mandana Chitsazan ${ }^{2}$; Mitra Chitsazan ${ }^{3, *}$; Negar Salehi ${ }^{3,4}$; Ahmad \\ Amin $^{3}$; Majid Maleki ${ }^{3}$; Nima Babaali ${ }^{3}$; Seifollah Abdi ${ }^{3}$; Maryam Mohsenian ${ }^{1}$ \\ ${ }^{1}$ Department of Cardiology, Rasoul-e-Akram Hospital, Iran University of Medical Sciences, Tehran, IR Iran \\ ${ }^{2}$ Shahid Beheshti University of Medical Sciences, Tehran, IR Iran \\ ${ }^{3}$ Rajaei Cardiovascular Medical and Research Center, Iran University of Medical Sciences, Tehran, IR Iran \\ 4 Michigan State University, Sparrow Health System, East Lansing, Michigan, USA \\ ${ }^{*}$ Corresponding author: Mitra Chitsazan, Rajaei Cardiovascular Medical and Research Center, Vali-Asr St., Niayesh Blvd, Tehran, IR Iran. Tel: +98-9122210385, Fax: +98-2122055594, \\ E-mail: mitra.chitsazan66@yahoo.com
}

Received: April 11, 2015; Revised: June 17, 2015; Accepted: July 19, 2015

\begin{abstract}
Background: The crucial role of inflammation in the development and progression of atherosclerosis has been previously described. However, there is insufficient data available to demonstrate the changes in high sensitivity C-reactive protein (hs-CRP) during clopidogrel therapy.

Objectives: In the present study, we aimed to assess the changes in the inflammatory marker of coronary heart disease, i.e., hs-CRP during clopidogrel therapy, in patients undergoing percutaneous coronary intervention (PCI). We also evaluated the anti-inflammatory effects of clopidogrel, if any, in different groups of patients.

Patients and Methods: The study population included 650 consecutive patients who underwent elective, urgent, or emergent PCI. Patients received a 300-mg loading dose of clopidogrel (Plavix ${ }^{\circledR}$ )and aspirin either 24 hours before the planned PCI, or immediately before the procedure in patients with urgent or emergent PCI, followed by a 75-mg daily maintenance dose for up to 12 weeks. At the end of the $12^{\text {th }}$ week, hs-CRP was re-assessed.

Results: Six hundred-fifty patients including 386 (59.4\%) male and 264 (40.6\%) female subjects were enrolled in the study. The mean hsCRP level was $15.36 \pm 9.83 \mathrm{mg} / \mathrm{L}$ with a median of $14 \mathrm{mg} / \mathrm{L}$ (interquartile range 8 to $19.6 \mathrm{mg} / \mathrm{L}$ ). Female, hypertensive, diabetic, and nonsmoking patients had higher reductions in hs-CRP in response to clopidogrel therapy compared to male, non-hypertensive, non-diabetic and smoker patients, respectively (all $\mathrm{P}<0.005$ ). The changes in the hs-CRP levels were also statistically different in patients with various index events before $\mathrm{PCI}(\mathrm{P}<0.001)$. No significant differences were observed in the mean reduction of hs-CRP between the patients without stent implantation and those with bare metal or drug-eluting stents $(\mathrm{P}=0.07)$, respectively.

Conclusions: We found that the use of clopidogrel in patients undergoing PCI had favorable effects on the suppression of hs-CRP. This effect appears to be heightened and more apparent in some group of patients with co-morbidities such as diabetes and hypertension.
\end{abstract}

Keywords: C-Reactive Protein; Clopidogrel; Coronary Artery Disease; Percutaneous Coronary Intervention

\section{Background}

The crucial role of inflammation in the development and progression of atherosclerosis has been previously described in detail $(1,2)$. Rather than being a simple marker, high-sensitivity C-reactive protein (hsCRP) acts as a regulator of many of these inflammatory pathways (3).

Epidemiologic studies have reported that hs-CRP levels add prognostic information for all levels of LDL cholesterol and at all levels of the Framingham risk score (4-6). A vast majority of prospective studies have also proven that the baseline levels of hs-CRP serves as a potent predictor of cardiovascular risk in apparently healthy individuals (4-16), as well as after adjustment for traditional and "novel" risk factors such as homocysteine and lipoprotein (a) (8-13). These studies all signify the importance of hs-CPP in atherosclerotic heart disease and indicate that hs-CRP is a critical participant in the atherothrombotic process.

Aspirin and statins have been previously shown to effectively decrease the plasma levels of hs-CRP. However, there are insufficient data on the changes of high sensitivity C-reactive protein during clopidogrel therapy.

\section{Objectives}

In the present study, we aimed to assess the changes of inflammatory marker of coronary heart disease, i.e., hsCRP, during clopidogrel therapy in patients undergoing percutaneous coronary intervention (PCI). We also evaluated the anti-inflammatory effects of clopidogrel, if any, in different groups of patients.

Copyright (C) 2016, Rajaie Cardiovascular Medical and Research Center, Iran University of Medical Sciences. This is an open-access article distributed under the terms of the Creative Commons Attribution-NonCommercial 4.0 International License (http://creativecommons.org/licenses/by-nc/4.0/) which permits copy and redistribute the material just in noncommercial usages, provided the original work is properly cited. 


\section{Patients and Methods}

\subsection{Patient Population}

The study population included 650 consecutive patients who underwent PCI in the catheterization laboratories of three referral hospitals (Pars General Hospital, Day General Hospital, and the Rajaie Cardiovascular Medical and Research Center) in Tehran, Iran from April 2010 to December 2012. Patients were eligible for enrollment if they had moderate- to high-risk unstable angina (UA/NSTEMI), ST-segment elevation MI (STEMI) with coronary anatomy known to be suitable for PCI (primary PCI), or if they were scheduled to undergo elective PCI without experiencing any type of acute coronary syndromes (with stable angina). Patients with conduction or rhythm abnormalities (bundle branch block, idioventricular rhythm, etc.), early coronary angiography due to recurrent ischemia or failed thrombolysis, treatment with aspirin or thienopyridines, any contraindications to aspirin or clopidogrel, past history of MI or coronary revascularization, presence of clinically assessed heart failure (Killip II/III) or cardiogenic shock, hepatic or renal failure (serum creatinine $>2.5 \mathrm{mg} / \mathrm{dL}$ ) and thrombocytopenia $\left(<100.000 / \mathrm{mm}^{3}\right)$, were excluded from the study. Patients with ongoing inflammatory disease or malignant or infective disorders were also excluded. The institutional review board approved the study protocol and patients signed a written informed consent prior to study participation.

\subsection{Blood Sampling}

Venous blood samples were obtained in the fasting state and before administration of the loading dose of clopidogrel in patients with planned PCI and immediately before PCI and administration of the loading dose of clopidogrel in patients with urgent or emergent PCI. Serum samples were frozen at $-70^{\circ} \mathrm{C}$ until analysis. Biochemical and hematological parameters were measured with Olympus AU600 autoanalyzer (Olympus Optical Co., Ltd., Schimatsu-Mishima, Japan) and Bayer Advia 120 Cell CBC Counter Hematologia autoanalyzer (Bayer Advia 120 CBC counter, NJ, USA). High-sensitivity CRP levels were determined by a chemiluminescent immunometric assay (Immulite; DPC, Los Angeles, USA) with a limit of quantification of $0.1 \mathrm{mg} / \mathrm{L}$, and an intraassay and interassay coefficient of variation of $2.09 \%$ and $5.88 \%$, respectively.

\subsection{Clopidogrel Administration}

Patients received a 300-mg loading dose of clopidogrel (Plavix ${ }^{\circledR}$ ) and aspirin (300 mg PO) either 24 hours before the planned PCI or immediately before the procedure in patients with urgent or emergent PCI, followed by 75-mg daily maintenance dose, for up to 12 weeks (17). At the end of the $12^{\text {th }}$ week, the hs-CRP was reassessed. A decision re- garding the continuation of clopidogrel therapy and duration of therapy beyond the study period was prepared for each patient, individually, based on the current treatment guidelines (17). All patients received daily aspirin and statin therapy based on the guideline (17).

\subsection{Statistical Analysis}

All analyses were conducted using IBM SPSS Statistics 19 for Windows (IBM Inc., Armonk, NY, USA). All data were initially analyzed using the Kolmogorov-Smirnov test to assess for normality. Quantitative variables were presented as means \pm standard deviation (SD) for normally distributed variables and as median (interquartile range, IQR) for variables without normal distribution. Categorical data were presented as numbers and percentages. Categorical data were compared with the chi-square test, while quantitative data were compared with Student's ttest, the Mann-Whitney and Kruskal Wallis tests, as appropriate. All P-values were two-tailed and a P value $<0.05$ was considered statistically significant.

\section{Results}

\subsection{Patient Characteristics}

Six hundred-fifty patients including 386 (59.4\%) male and 264 (40.6\%) female subjects were enrolled in the study. The average age was $63.75 \pm 8.37$ years. Baseline demographic characteristics of patients based on the hsCRP tertiles, are illustrated in Table 1 . The mean hs-CRP level was $15.36 \pm 9.83 \mathrm{mg} / \mathrm{L}$, with a median of $14 \mathrm{mg} / \mathrm{L}$ (interquartile range 8 to $19.6 \mathrm{mg} / \mathrm{L}$ ). Tertiles for hs-CRP were $<10,10$ to 17 , and $>17 \mathrm{mg} / \mathrm{L}$.

No significant differences in age, gender, body mass index (BMI), hypertension, diabetes mellitus, smoking, intake of beta-blocker or ACEI/ARB medications (all P > 0.05 ) were observed between the patients in the lowest and those in the 2 higher hs-CRP tertiles. However, patients in the lowest hs-CRP tertile were more likely to take statin than patients in the 2 highest hs-CRP tertiles $(\mathrm{P}<$ 0.001).

\subsection{Association of hs-CRP With the Index Event}

Baseline median serum levels of hs-CRP were $6.9 \mathrm{mg} / \mathrm{L}$ (IQR, 4.3 - $11 \mathrm{mg} / \mathrm{L}), 16 \mathrm{mg} / \mathrm{L}$ (IQR, 12 - $19.9 \mathrm{mg} / \mathrm{L}), 19 \mathrm{mg} / \mathrm{L}$ (IQR, 14.5 - $23.4 \mathrm{mg} / \mathrm{L}$ ) and $29.9 \mathrm{mg} / \mathrm{L}(26$ - 41) $\mathrm{mg} / \mathrm{L}$ in patients with elective, unstable angina, NSTEMI, and STEMI index events, respectively, with statistically significant differences $(\mathrm{P}<0.001)$.

\subsection{Association of hs-CRP With the Index Procedure}

Patients who underwent multivessel PCI had significantly higher baseline serum levels of hs-CRP compared to patients with single vessel PCI (11.5; IQR, 7 - 16 versus 15.5; IQR, 8.7 - 22 mg/L, P < 0.001). 
Hajsadeghi S et al.

\begin{tabular}{|c|c|c|c|}
\hline Variable & Tertile I, $<10 \mathrm{mg} / \mathrm{L}(\mathrm{n}=\mathbf{2 2 3})$ & Tertile II, III, > $10 \mathrm{mg} / \mathrm{L}(\mathrm{n}=427)$ & P Values \\
\hline \multicolumn{4}{|l|}{ Clinical Characteristics } \\
\hline Age, y & $64.39 \pm 8.29$ & $63.22 \pm 8.56$ & 0.25 \\
\hline Male gender & $140(63)$ & $246(57.61)$ & 0.23 \\
\hline Body mass index, $\mathrm{kg} / \mathrm{m}^{2}$ & $26.98(24.01-31.18)$ & $26.96(24.7-30.14)$ & 0.50 \\
\hline Diabetes & $55(25)$ & $124(29)$ & 0.26 \\
\hline Hypertension & $44(20)$ & $111(26)$ & 0.75 \\
\hline Current smoking & $138(62)$ & $231(54)$ & 0.057 \\
\hline Dyslipidemia & $107(48)$ & $143(33.5)$ & 0.54 \\
\hline Clinical indication & & & $<0.001$ \\
\hline Elective & $175(78.5)$ & $71(16.7)$ & \\
\hline UA & $38(17)$ & $202(47.3)$ & \\
\hline NSTEMI & $10(4.5)$ & $111(26)$ & \\
\hline STEMI & 0 & $43(10)$ & \\
\hline Procedural characteristics & & & $<0.001$ \\
\hline One vessel disease & $94(42)$ & $119(28)$ & \\
\hline Multivessel disease & $129(58)$ & $308(72)$ & \\
\hline \multicolumn{4}{|l|}{ Medications } \\
\hline Beta blockers & $46(20)$ & $100(23.4)$ & 0.41 \\
\hline ACE inhibitors/ARBs & $43(19)$ & $55(13)$ & 0.30 \\
\hline Statins & $133(59.5)$ & $37(9)$ & $<0.001$ \\
\hline Calcium channel blockers & $4(1.8)$ & $12(2.8)$ & 0.65 \\
\hline
\end{tabular}

\subsection{Changes of hs-CRP After Clopidogrel Treatment}

After therapy, median serum level of hs-CRP significantly decreased from $14 \mathrm{mg} / \mathrm{L}$ (IQR, 8 - $19.6 \mathrm{mg} / \mathrm{L}$ ) at baseline to $3.8 \mathrm{mg} / \mathrm{L}(\mathrm{IQR}, 2.1-9 \mathrm{mg} / \mathrm{L})$ at the $12^{\text {th }}$ week $(\mathrm{P}<0.001)$. Female patients had higher reductions in hs-CRP levels in response to clopidogrel therapy compared to male patients $(\mathrm{P}=0.006)$. Moreover, hypertensive patients had higher reductions in hs-CRP levels compared to nonhypertensive patients $(\mathrm{P}=0.002)$. Diabetic patients also had higher reductions in hs-CRP levels after clopidogrel therapy $(\mathrm{P}=0.004)$. Non-smokers had higher reductions in hs-CRP levels compared to patients who smoked ( $\mathrm{P}=$ 0.001). Patients who had been taking statins before PCI also had lower reductions in hs-CRP levels compared to patients without statin use before PCI $(\mathrm{P}<0.001)$ while patients with and without ACEI/ARB intake had comparable reduction in hs-CRP levels $(\mathrm{P}=0.54)$. The changes in the hs-CRP levels were also statistically different in patients with various index events before PCI $(\mathrm{P}<0.001$, Figure 1). The percent of changes in hs-CRP levels from baseline to the $12^{\text {th }}$ week was $-58.5 \%,-62.34 \%,-54.86 \%$, and $-49.74 \%$ in patients with elective, UA, NSTEMI and STEMI index events, respectively $(\mathrm{P}=0.57)$. No significant differences were observed in the mean reduction of hs-CRP levels between patients without stent implantation and those with bare metal or drug-eluting stents $(P=0.07)$. The summaries of the changes in the different subgroups of patients are outlined in Tables 2 - 4 based on clinical traits, medication use, and periprocedural characteristics, respectively. Multivariate regression analysis was performed in order to identify predictors of change in hs-CRP (Table 4).

Figure 1. The Changes in the hs-CRP Levels Were Statistically Different in Patients With Various Index Events Before PCI.

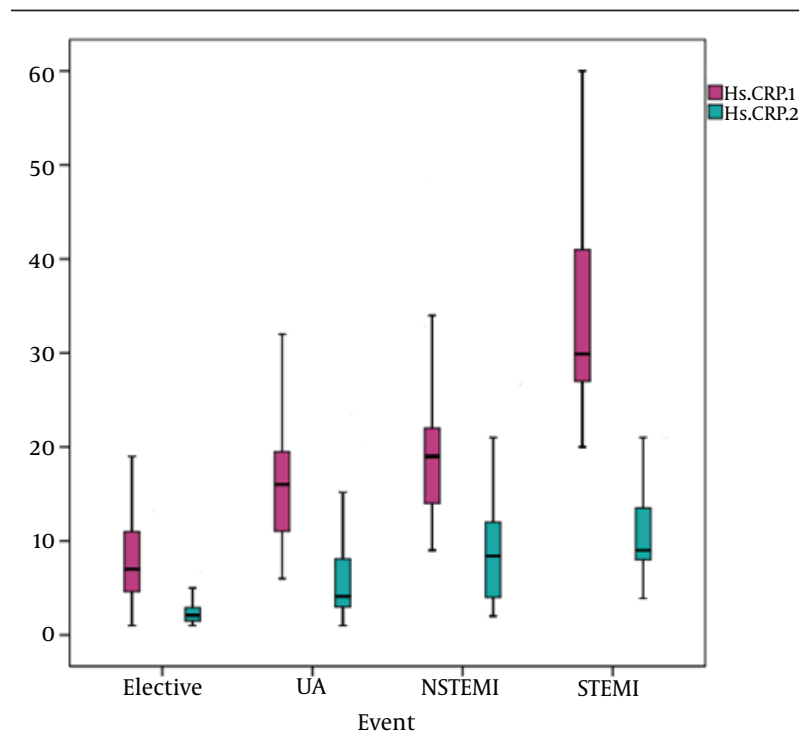

Hs-CRP 1 and 2: hs-CRP levels before and after treatment with clopidogrel, respectively. 
Hajsadeghi S et al.

\begin{tabular}{|c|c|c|c|c|c|}
\hline \multirow[t]{2}{*}{ Clinical Characteristic } & \multicolumn{5}{|c|}{ Hs-CRP } \\
\hline & BeforeTreatment & PValue & After Treatment & Change & P Value \\
\hline Gender & & 0.013 & & & 0.006 \\
\hline Male & $13.00(8.00-18.00)$ & & $3.60(2.10-7.00)$ & $-8.00(-12.00-4.90)$ & \\
\hline Female & $15.90(8.90-20.50)$ & & $3.40(2.10-9.00)$ & $-10.00(-14.00-5.40)$ & \\
\hline Hypertension & & 0.29 & & & 0.002 \\
\hline Yes & $14.00(9.00-19.97)$ & & $3.10(2.09-5.80)$ & $-10.00(-14.00-6.02)$ & \\
\hline No & $14.00(8.00-19.00)$ & & $3.60(2.10-8.32)$ & $-8.25(-13.00-4.70)$ & \\
\hline Diabetes & & 0.59 & & & 0.004 \\
\hline Yes & $13.65(8.97-19.00)$ & & $2.95(2.00-6.05)$ & $-9.95(-13.62-6.00)$ & \\
\hline No & $14.00(8.00-19.00)$ & & $3.90(2.10-8.40)$ & $-8.05(-13.35-4.70)$ & \\
\hline Smoking & & 0.004 & & & 0.001 \\
\hline Yes & $13.06(7.00-18.00)$ & & $3.10(2.10-7.00)$ & $-8.00(-12.57-4.70)$ & \\
\hline No & $15.20(9.00-21.00)$ & & $3.90(2.10-9.00)$ & $-9.90(-13.95-5.80)$ & \\
\hline
\end{tabular}

Table 3. The Effects of Clopidogrel on hs-CRP in Patients With Different Medications a

\begin{tabular}{|c|c|c|c|c|c|}
\hline \multirow[t]{2}{*}{ Medication } & \multicolumn{5}{|c|}{ Hs-CRP } \\
\hline & Before Treatment & P Value & After Treatment & Change & PValue \\
\hline Beta blocker & & 0.94 & & & 0.35 \\
\hline Yes & $14.00(8.90-17.90)$ & & $3.10(2.00-6.40)$ & $-9.30(-13.38-5.50)$ & \\
\hline No & $14.00(8.00-19.00)$ & & $3.60(2.10-8.30)$ & $-8.50(-13.40-5.00)$ & \\
\hline ACEI/ARB & & 0.57 & & & 0.54 \\
\hline Yes & $13.00(8.00-19.00)$ & & $3.00(2.00-6.70)$ & $-8.50(-12.95-5.00)$ & \\
\hline No & $14.90(8.90-19.00)$ & & $3.60(2.10-8.00)$ & $-8.90(-13.4-5.00)$ & \\
\hline Calcium channel blocker & & $<0.001$ & & & $<0.001$ \\
\hline Yes & $16.00(14.00-19.87)$ & & $4.10(3.00-7.47)$ & $-11.70(-14.00-8.37)$ & \\
\hline No & $13.00(7.00-19.00)$ & & $3.10(2.00-8.00)$ & $-8.00(-12.97-4.61)$ & \\
\hline Statins ${ }^{b}$ & & $<0.001$ & & & $<0.001$ \\
\hline Yes & $6.15(4.08-10.30)$ & & $2.10(1.30-3.00)$ & $-4.68(-7.20-2.19)$ & \\
\hline No & $16.00(11.00-21.00)$ & & $4.15(2.90-9.50)$ & $-10.20(-14.00-6.90)$ & \\
\hline
\end{tabular}

Table 4. The Effects of Clopidogrel on hs-CRP in Patients With Different Periprocedural Characteristics ${ }^{a}$

\begin{tabular}{|c|c|c|c|c|c|}
\hline \multirow[t]{2}{*}{ Periprocedural Characteristics } & \multicolumn{5}{|c|}{ Hs-CRP } \\
\hline & Before Treatment & P Value & After Treatment & Change & P Value \\
\hline Clinical indication & & $<0.001$ & & & $<0.001$ \\
\hline STEMI & $7.00(4.53-11.00)$ & & $2.10(1.50-2.90)$ & $-4.90(-8.00-2.89)$ & \\
\hline Elective & $16.00(11.01-19.50)$ & & $4.10(3.00-8.15)$ & $-10.80(-14.30-7.10)$ & \\
\hline UA & $19.00(14.00-22.00)$ & & $8.40(4.00-12.00)$ & $-10.50(-14.00-6.00)$ & \\
\hline NSTEMI & $29.90(26.00-41.00)$ & & $9.00(8.00-14.00)$ & $-19.60(-24.00-17.00)$ & \\
\hline Procedural characteristics & & $<0.001$ & & & $<0.001$ \\
\hline One vessel & $11.00(7.00-16.00)$ & & $3.10(2.00-5.30)$ & $-7.00(-10.6-4.80)$ & \\
\hline Multivessel & $15.50(9.00-21.00)$ & & $3.80(2.10-9.00)$ & $-10.00(-14.00-5.10)$ & \\
\hline Stent implantation & & 0.27 & & & 0.07 \\
\hline No & $13.00(8.95-16.00)$ & & $3.90(2.10-8.55)$ & $-7.80(-10.70-5.00)$ & \\
\hline BMS & $13.40(9.00-18.00)$ & & $3.20(2.10-6.30)$ & $-8.10(-12.90-6.00)$ & \\
\hline DES & $15.00(8.00-19.90)$ & & $3.55(2.00-8.20)$ & $-9.35(-13.90-4.87)$ & \\
\hline
\end{tabular}


Hajsadeghi S et al.

\begin{tabular}{|c|c|c|c|c|}
\hline \multirow[t]{2}{*}{ Predictor } & \multicolumn{2}{|c|}{ Unstandardized Coefficients } & \multirow{2}{*}{$\begin{array}{c}\text { Standardized Coefficients } \\
\text { Beta }\end{array}$} & \multirow[t]{2}{*}{ P Values } \\
\hline & B & Standard Error & & \\
\hline Constant & -6.905 & 1.293 & & $<0.001$ \\
\hline Statin & -4.824 & 0.175 & 0.718 & $<0.001$ \\
\hline DM & 1.554 & 0.306 & 0.132 & $<0.001$ \\
\hline CCB & 2.008 & 0.392 & 0.134 & $<0.001$ \\
\hline ACEI/ARB & 0.847 & 0.374 & 0.058 & 0.024 \\
\hline Smoking & -0.467 & 0.222 & -0.054 & 0.036 \\
\hline
\end{tabular}

a abbreviations: ACEI, angiotensin converting enzyme inhibitor; ARB, angiotensin II receptor blocker; CCB, calcium channel blocker, and DM, diabetes mellitus.

\section{Discussion}

The results of the current study should be discussed in three separate sections. First, we tried to find risk factors for a higher baseline hs-CRP level in patients undergoing elective, urgent or emergency PCI. Unsurprisingly, hs-CRP levels varied significantly in patients with various types of acute coronary syndromes. High risk events were associated with higher baseline hs-CRP level. Moreover, patients who underwent multi-vessel PCI had higher hsCRP levels compared to patients with one-vessel PCI, indicating that more extended coronary artery disease may cause higher levels of hs-CRP.

Second, we assessed changes in the hs-CRP levels in these patients following the administration of clopidogrel for 12 weeks. The main finding was that serum levels of hs-CRP significantly decreased throughout the study period. The anti-inflammatory properties of clopidogrel have been revealed by some of the earlier studies. A reduction in the number of platelet-leukocyte interactions has been reported previously $(18,19)$. It was also demonstrated that clopidogrel decreased the periprocedural increase in hs-CRP levels in patients undergoing PCI by $65 \%$ (20). Quinn and his colleagues also demonstrated that clopidogrel pretreatment reduced platelet inflammatory marker expression in patients undergoing PCI (21). However, the present study is one of the few studies, at least to best of our knowledge, which assessed the longterm anti-inflammatory effects of clopidogrel in patients undergoing PCI.

Third, we performed several subgroup analyses in order to identify patients with higher reductions in hs-CRP from baseline with clopidogrel therapy. The subgroup analysis demonstrated that although the baseline hsCRP and the magnitude of changes over the study period significantly differed between the patients with various index events, the percentages of changes in the hs-CRP from the baseline values were comparable in all groups, and the decrease in hs-CRP levels was proportional to the baseline values. Although patients with more severe cardiovascular events had higher hs-CRP levels and higher reductions over 3 months, they had the same percentage of reduction in hs-CRP levels as the patients with lower risks for events. This suggests that there are no specific types of cardiovascular events, which benefit more from the clopidogrel therapy, according to its anti-inflammatory effects.

In several studies, statins have been demonstrated to decrease hs-CRP levels and coronary events independently of serum lipid concentrations $(4,11)$. In our study population, 170 (26\%) patients were taking statins before PCI. These patients were more likely to be in the lower tertile of the baseline hs-CRP (78\%) compared to the 2 higher hsCRP tertiles (22\%). For the patients that had not previously been on statins, statins were started immediately after PCI, according to the current guidelines (17). We demonstrated that patients who were on statins before PCI had lower reductions in hs-CRP during clopidogrel administration compared to those who started statins after PCI. However, it is possible that the lower reductions in hsCRP levels in patients taking statins arises from the lower baseline hs-CRP rather than the lower efficacy of clopidogrel in these patients. Aspirin also has anti-inflammatory activities (2). However, since all of the patients received aspirin during the study period, it should not be considered as a confounding factor for the reduction of hs-CRP. Although patients taking ACEIs were more likely to be present in the lower tertile of the baseline hs-CRP, there were no significant differences in the reduction of hs-CRP levels in patients who were taking ACEI during clopidogrel therapy, compared to who were not doing so.

Although both groups had comparable baseline hs-CRP levels, the results of our study demonstrated that diabetic patients had higher decreases in hs-CRP levels compared to non-diabetic patients. This finding may focus the attention on the potentially higher beneficial effects of clopidogrel in diabetic patients and should herald questions in order to clearly define the anti-inflammatory role of clopidogrel in these patients, even in the absence of established coronary heart disease. Moreover, it was demonstrated previously that elevated hs-CRP levels in diabetic patients could predict cardiovascular mortal- 
ity independently of traditional risk factors (21). This may provide further rationale for future investigations on the beneficial effects of clopidogrel in diabetic patients, regardless of the concomitant coronary heart disease. Additionally, despite statistically similar baseline hs-CRP values, hypertensive patients also had higher reductions in hs-CRP compared to non-hypertensive patients. We also observed higher reductions in hs-CRP levels in nonsmokers comparing to smoker patients. Since non-smokers had statistically lower baseline hs-CRP levels as well as similar percentages of change in hs-CRP levels compared to smoker patients, we were unable to definitively determine if smoking alters the anti-inflammatory effects of clopidogrel. Further studies are needed to confirm these results.

Multivariate regression analysis also demonstrated that statins, DM, CCB, ACEI, and smoking were significant independent predictors of the changes in serum hs-CRP levels over the study period. The major limitation of the present study was the lack of a control group. However, since clopidogrel is a class I recommendation for the long-term treatment of patients undergoing PCI, especially in patients with stent implantation, it was impossible has to have a control group from an ethical standpoint.

In conclusion, we learned that the use of clopidogrel in patients undergoing PCI had favorable effects on the suppression of hs-CRP levels. This effect appears to be heightened in some group of patients with co-morbidities such as diabetes and hypertension.

\section{Acknowledgements}

We gratefully acknowledge the generous assistance by Ms. Mir Ahsani from the catheterization laboratory of the Pars General Hospital.

\section{Authors' Contributions}

Shokoufeh Hajsadeghi contributed to the study concept and design, critical revision and approval of the manuscript. Negar Salehi, Ahmad Amin, Majid Maleki, Nima Babaali, Seifollah Abdi, and Maryam Mohsenian contributed to the data collection, critical revision, and approval of the manuscript. Mitra Chitsazan and Mandana Chitsazan also contributed to the study concept and design, data collection, analysis and interpretation, statistics, drafting, critical revision, and approval of the manuscript.

\section{References}

1. Ross R. Atherosclerosis--an inflammatory disease. $N$ Engl J Med. 1999;340(2):115-26.

2. Libby P, Ridker PM, Maseri A. Inflammation and atherosclerosis. Circulation. 2002;105(9):1135-43.

3. Torres JL, Ridker PM. Clinical use of high sensitivity C-reactive protein for the prediction of adverse cardiovascular events. Curr Opin Cardiol. 2003;18(6):471-8.
4. Ridker PM, Rifai N, Rose L, Buring JE, Cook NR. Comparison of C-reactive protein and low-density lipoprotein cholesterol levels in the prediction of first cardiovascular events. $N$ Engl J Med. 2002;347(20):1557-65.

5. Ridker PM, Buring JE, Cook NR, Rifai N. C-reactive protein, the metabolic syndrome, and risk of incident cardiovascular events: an 8-year follow-up of 14719 initially healthy American women. Circulation. 2003;107(3):391-7.

6. Albert MA, Glynn RJ, Ridker PM. Plasma concentration of C-reactive protein and the calculated Framingham Coronary Heart Disease Risk Score. Circulation. 2003;108(2):161-5.

7. Ridker PM. High-sensitivity C-reactive protein: potential adjunct for global risk assessment in the primary prevention of cardiovascular disease. Circulation. 2001;103(13):1813-8.

8. Ridker PM, Cushman M, Stampfer MJ, Tracy RP, Hennekens CH. Inflammation, aspirin, and the risk of cardiovascular disease in apparently healthy men. NEngl J Med.1997;336(14):973-9.

9. Ridker PM, Hennekens $\mathrm{CH}$, Buring JE, Rifai N. C-reactive protein and other markers of inflammation in the prediction of cardiovascular disease in women. N Engl J Med. 2000;342(12):836-43.

10. Ridker PM, Rifai N, Clearfield M, Downs JR, Weis SE, Miles JS, et al. Measurement of C-reactive protein for the targeting of statin therapy in the primary prevention of acute coronary events. $N$ Engl J Med. 2001;344(26):1959-65.

11. Ridker PM, Stampfer MJ, Rifai N. Novel risk factors for systemic atherosclerosis: a comparison of C-reactive protein, fibrinogen, homocysteine, lipoprotein(a), and standard cholesterol screening as predictors of peripheral arterial disease. JAMA. 2001;285(19):2481-5.

12. Koenig W, Sund M, Frohlich M, Fischer HG, Lowel H, Doring A, et al. C-Reactive protein, a sensitive marker of inflammation, predicts future risk of coronary heart disease in initially healthy middle-aged men: results from the MONICA (Monitoring Trends and Determinants in Cardiovascular Disease) Augsburg Cohort Study, 1984 to 1992. Circulation. 1999;99(2):237-42.

13. Danesh J, Whincup P, Walker M, Lennon L, Thomson A, Appleby $P$, et al. Low grade inflammation and coronary heart disease: prospective study and updated meta-analyses. BMJ. 2000;321(7255):199-204.

14. Pradhan AD, Manson JE, Rossouw JE, Siscovick DS, Mouton CP, Rifai N, et al. Inflammatory biomarkers, hormone replacement therapy, and incident coronary heart disease: prospective analysis from the Women's Health Initiative observational study. JAMA. 2002;288(8):980-7.

15. Albert CM, Ma J, Rifai N, Stampfer MJ, Ridker PM. Prospective study of C-reactive protein, homocysteine, and plasma lipid levels as predictors of sudden cardiac death. Circulation. 2002;105(22):2595-9.

16. Mendall MA, Strachan DP, Butland BK, Ballam L, Morris J, Sweetnam PM, et al. C-reactive protein: relation to total mortality, cardiovascular mortality and cardiovascular risk factors in men. Eur Heart J. 2000;21(19):1584-90.

17. Kolh P, Alfonso F, Collet JP, Cremer J, Falk V, StephanWindecker. et al. 2014 ESC/EACTS guidelines on myocardial revascularization. Rev Esp Cardiol (Engl Ed). 2015;68(2):144

18. Storey RF, Judge HM, Wilcox RG, Heptinstall S. Inhibition of ADPinduced P-selectin expression and platelet-leukocyte conjugate formation by clopidogrel and the P2Y12 receptor antagonist ARC69931MX but not aspirin. Thromb Haemost. 2002;88(3):488-94.

19. Klinkhardt U, Graff J, Harder S. Clopidogrel, but not abciximab, reduces platelet leukocyte conjugates and P-selectin expression in a human ex vivo in vitro model. Clin Pharmacol Ther 2002;71(3):176-85.

20. Vivekananthan DP, Bhatt DL, Chew DP, Zidar FJ, Chan AW, Moliterno DJ, et al. Effect of clopidogrel pretreatment on periprocedural rise in C-reactive protein after percutaneous coronary intervention. Am J Cardiol. 2004;94(3):358-60.

21. Quinn MJ, Bhatt DL, Zidar F, Vivekananthan D, Chew DP, Ellis SG et al. Effect of clopidogrel pretreatment on inflammatory marker expression in patients undergoing percutaneous coronary intervention. Am J Cardiol. 2004;93(6):679-84. 